\title{
The Method of Allocation Centers in SECOND KIND FUZZY GRAPHS WITH THE LARGEST VITALITY DEGREE
}

\author{
Alexander Bozhenyuk, Stanislav Belyakov and Margarita Knyazeva
}

\begin{abstract}
The problem of optimal allocation of service centers is considered in this paper. It is supposed that the information received from GIS is presented like second kind fuzzy graphs. Method of optimal location as method of finding vitality fuzzy set of second kind fuzzy graph is suggested. Basis of this method is building procedure of reachability matrix of second kind fuzzy graph in terms of reachability matrix of first kind fuzzy graph. This method allows solving not only problem of finding of optimal service centers location but also finding of optimal location $k$-centers with the greatest degree and selecting of service center numbers. The algorithm of the definition of vitality fuzzy set for second kind fuzzy graphs is considered. The example of finding optimum allocation centers in second kind fuzzy graph is considered too.
\end{abstract}

Keywords—second kind fuzzy graph; service centers; vitality fuzzy set

\section{INTRODUCTION}

$\mathrm{T}_{\text {im }}^{\mathrm{HE}}$ HE worldwide expansion and diversified implementation of geographic information systems (GIS) is largely due to the need to improve information systems that support decision-making. Application spheres of GIS are huge, thus geoinformation technologies become leaders in information retrieval, display, analytical tools and decision support $[1,2]$.

However, geographic data are often associated with significant uncertainty. Errors in data that are often used without considering their inherent uncertainties lead to a high probability of obtaining information of doubtful value. Uncertainty presents throughout the process of geographical abstraction: from acquiring data to using them [3].

Data modeling [4] is the process of abstraction and generation of real forms of geographic data. This process provides a conceptual model of the real world. It is doubtful that the geographical complexity can be reduced in models of perfect accuracy. So, the imminent contradiction between the real world and the model is the inaccuracy and uncertainty that can lead to the wrong decision making.

Academy for Engineering and Technologies, Southern Federal University, Taganrog, Russia
Allocation of centers [5] is the optimization task, effectively solved by GIS. This problem includes the tasks of optimum allocation of extremely important services, such as hospitals, police stations, fire brigades etc. In some tasks, the optimality criterion can considered as distance minimization (travel time) from the service center to the most remote service point, therefore, the problem is in optimization of the "worst case" [6]. At the same time, the information, presented in GIS, can be approximate or insufficiently reliable [7].

Information inaccuracy, as well as parameter uncertainty, uncertainty that usually happens in decisionmaking process, uncertainty caused by environmental influence - are the examples of fuzziness concerning of servicing centers allocation problems.

For example, information inaccuracy can consider imprecise measurement of distance between serving facilities, such as distance measure, the measuring accuracy equaling in several centimeters or meters may appear.

Parameter measuring process is connected with the fact that some characteristics are qualitative and don't have numerical equivalents: quality of some part of a route can't be measured but it can be described like "good", "bad", "the worst" and etc. Besides, some objects can change parameters by their own, for example, sizes of waters change naturally or its borders are indistinct.

Uncertainty in decision-making process supposes imprecise data about targets and some parameters that can't be determined exactly or it allows variations in the certain range. Implementation of fuzzy theory allows to describe reality more adequate and permits to find more suitable decision. Uncertainty caused by environmental influence supposes influence of some factors on parameters of objects or relations that changes their values.

Some types of inaccuracy of parameters measurement of objects with complex structure and impact of external factors on parameters that are taking into account for 
optimization are usually considered. Then location problems are solved under uncertain conditions [8].

We consider that a certain way system has $n$ stations. There are $k$ service centres, which may be placed into these stations. Each centre can serve several stations. The degree of a service station by a centre depends on a route which connects them. It is necessary for the given number of centers to define the places of their best allocation. In other words, it is necessary to define the places of $k$ centers into $n$ stations so that the «control» of all territory (all stations) is carried out with the greatest possible degree of service.

In works [9-11], this problem has been considered as a problem of allocation centers on fuzzy graphs of the first kind $[12,13]$, i.e. graphs whose set of vertices is nonfuzzy set, and set of edges is fuzzy set. Sometimes, however, adequate model of such allocation is fuzzy graph is a more general form [14, 15], in which a set of vertices and a set of edges are fuzzy sets. Such a graph in the work [16] was named the fuzzy graph of the second kind.

The approach to finding the best centers allocation on the fuzzy graph of the second kind is considered in this paper.

\section{BASIC CONCEPTS AND DEFINITIONS}

For choosing of the best location of service centers we can use the notion of conjunctive strength and the reachability degree of vertex. In this case we will compare all of the movement paths among themselves and find routes with a maximal reachability degree.

Let $\tilde{G}=(\tilde{X}, \tilde{U})$ is a second kind fuzzy graph [16]. Here a set $\tilde{X}=\left\{<\mu_{X}(x) / x>\right\}$, is the fuzzy set of vertices, defined on set $X, \quad|X|=n, \quad$ and $\tilde{U}=\left\{<\mu_{U}\left(x_{i}, x_{j}\right) /\left(x_{i}, x_{j}\right)>\right\}, x_{i}, x_{j} \in X$ is fuzzy set of directed edges. Here $\mu_{\mathrm{X}}(x) \in[0,1]$ - membership function for vertex $x, \mu_{U}\left(x_{i}, x_{j}\right) \in[0,1]$ - membership function for edge $\left(x_{i}, x_{j}\right)$.

A path of fuzzy graph $l\left(x_{i}, x_{j}\right)$ is called a directed sequence of fuzzy edges from vertex $x_{i}$ to vertex $x_{j}$, in which the final vertex of any edge is the first vertex of the following edge [12, 13].

A conjunctive strength of second kind fuzzy graph is defined by expression:

Copyright (C) Authors

$$
\mu_{l}\left(x_{i}, x_{j}\right)=\sum_{<x_{k}, x_{t}>\in l\left(x_{i}, x_{j}\right)}^{\&} \mu_{U}\left(x_{k}, x_{t}\right) \& \mu_{\substack{X \\ x_{t} \neq x_{i} \\ x_{i} \neq x_{j}}}\left(x_{t}\right) .
$$

In other words, the conjunctive strength of second kind fuzzy graph is defined by the smallest value of membership functions of vertices and edges that are included in this path, except for the first and last vertices of the path.

Let $L$ is a family of second kind fuzzy graph paths from vertex $x_{i}$ to vertex $x_{j}$. Then the value $\gamma\left(x_{i}, x_{j}\right)=\max _{l \in L}\left\{\mu_{l}\left(x_{i}, x_{j}\right)\right\}$ defines the reachability degree of vertex $x_{j}$ from vertex $x_{i}$.

The task of the best allocation of centers on the fuzzy graph can be limited to the problem of finding a subset of vertices $Y$, which all the other vertices $X / Y$ of the fuzzy graph achievable from with the greatest reachability degree. There are three strategies of the selection of vertices $Y$ [11]. - We "go" from each vertex of subset $X / V$, and arrive at a vertex of $V$;

- We "come out" of any of the vertices of $V$, and reach all vertices of subset $X / \mathrm{V}$;

- We "come out" of any of the vertices of $V$, reach all vertices of subset $X / V$ and come back.

In this paper, the third strategy is considered.

Let $k$ is service centers $(k<n)$, placed in the vertices of subset $Y,|Y|=k, Y \subset X$, and $\gamma\left(x_{i}, x_{j}\right)$ is reachability degree of vertex $x_{j}$ from vertex $x_{i}$.

Definition 1. Value

$$
V_{\tilde{G}}(Y)=\underset{\forall x_{j} \in X \backslash Y}{\&}\left(\underset{\forall x_{i} \in Y}{\vee} \gamma\left(\mathrm{x}_{\mathrm{i}}, \mathrm{x}_{\mathrm{j}}\right) \& \gamma\left(\mathrm{x}_{\mathrm{j}}, \mathrm{x}_{\mathrm{i}}\right)\right)
$$

is a vitality degree of fuzzy graph $\widetilde{\mathrm{G}}$ which is served by $k$ centers from vertex set $Y$.

Vitality degree $\mathrm{V}_{\tilde{\mathrm{G}}}(Y)$ determines the minimax strong connectivity value between each vertex from set $X \backslash Y$ and a center from set $Y$.

In other words, one can "leave" the vertex of subset $Y$, "reach" any vertex of the graph, "serve" it, return to the "initial" vertex while the conjunctive strength of the route will not be less than value $V_{\widetilde{G}}(Y)$. 
It is clear that value $V_{\widetilde{G}}(Y) \in[0,1]$ depends either on the number of centers $k$, or the allocation of the centers on the vertices of graph $\widetilde{G}$ (i.e. on the choice of set $Y$ ).

Thus, the problem of the allocation of $k$ service centers $(k<n)$ in fuzzy graph $\widetilde{G}$ is reduced to determining such a subset of vertices $Y \subset X$, that value of vitality degree $V_{\widetilde{G}}(Y)$ reaches its maximum value, that is value $V_{\tilde{G}}(k)=\max _{\substack{\forall Y \subset X \\|Y|=k}}\left\{V_{\tilde{G}}(Y)\right\}$.

\section{Definition 2. Fuzzy set}

$$
\tilde{\mathrm{V}}_{\tilde{\mathrm{G}}}=\left\{<V_{\tilde{G}}(1) / 1>,<V_{\tilde{G}}(2) / 2>, \ldots,<V_{\tilde{G}}(n) / n>\right\},
$$

defined on vertex set $X$, is called a fuzzy set of vitality of graph $\tilde{G}=(X, \tilde{U})$. Fuzzy set of vitality $\tilde{\mathrm{V}}_{\tilde{\mathrm{G}}}$ determines the greatest vitality degrees of graph $\widetilde{G}$ if it is served by 1 , $2 \ldots n$ centers.

Values $\tilde{\mathrm{V}}_{\tilde{\mathrm{G}}}(k)(1 \leq k \leq n)$ signify that we can place $k$-centers in graph $\tilde{G}$ so that there is a route from at least one center to any vertex of graph $\tilde{G}$ and back. The conjunctive strength of the graph will be not less than $\tilde{\mathrm{V}}_{\widetilde{\mathrm{G}}}(k)$.

The fuzzy setoff vitality is a fuzzy invariant of a fuzzy graph. It determines the highest degree of the reachability of the vertices for any given number of service centers.

\section{METHOD FOR FINDING OF VITALITY FUZZY SET}

We will consider the method of finding a family of all service centers with the largest vitality degree for second kind fuzzy graph.

In $[10,11]$ was considered the method for first kind fuzzy graph. Let $Y$ be a subset of the vertices of fuzzy graph $\tilde{G}=(X, \tilde{U})$ in which the service centers are located and the vitality degree equals to $V$. Therefore, one of the two conditions for any vertex $x_{i} \in X$ can be satisfied:

a) vertex $x_{i}$ belongs to set $Y$;

b) there is vertex $x_{j}$ that belongs to set $Y$ and inequalities $\gamma\left(x_{i}, x_{j}\right) \geq V \quad$ and $\quad \gamma\left(x_{j}, x_{i}\right) \geq V \quad$ are encountered.

Using the notation quantifier form we can get the truth of the following formula:

Copyright (C) Authors

$$
\begin{aligned}
& \left(\forall \mathrm{x}_{\mathrm{i}} \in X\right)\left[x_{i} \in Y \vee\left(\exists x_{j}\right)\right. \\
& \left.\left(x_{j} \in Y \& \gamma\left(x_{i}, x_{j}\right) \geq V \& \gamma\left(x_{j}, x_{i}\right) \geq V\right)\right] .
\end{aligned}
$$

To each vertex $x_{i} \in X$ we assign Boolean variable $p_{i}$ that takes value 1 , if $x_{i} \in Y$ and 0 otherwise. We assign the fuzzy variable $\xi_{j i}=\chi\left(x_{j}, x_{i}\right)$ for the proposition $\chi\left(x_{j}, x_{i}\right) \geq V$. Passing from the quantifier form of proposition (3) to the form in terms of logical operations, we obtain a true logical proposition:

$$
\Phi_{V}=\underset{i}{\&}\left(p_{i} \vee\left(\bar{p}_{i} \rightarrow\left(\underset{j}{\vee}\left(p_{j} \& \gamma_{j i}\right)\right)\right)\right)
$$

Taking into account the interrelation between the implication operation and disjunction operation, we receive:

$$
\Phi_{\mathrm{V}}=\underset{i=1, n}{\&}\left(p_{i} \vee \underset{j=1, n}{\vee}\left(p_{j} \& \xi_{i j} \& \xi_{i j}\right)\right)
$$

Supposing $\xi_{i i}=1$ and considering that the equality $p_{i} \vee \underset{j}{\vee} p_{i} \& \xi_{i j}=\underset{j}{\vee} p_{j} \xi_{i j}$ is true for any vertex $x_{i}$, we finally obtain:

$$
\Phi_{V}=\underset{i=1, n}{\&} \underset{j=1, n}{\vee}\left(\xi_{i j} \& \xi_{j i} \& p_{j}\right)
$$

We open the parentheses in the expression (4) and reduce the similar terms by following rules (5).

$$
a \vee a \& b=a ; a \& b \vee a \& \bar{b}=a ; \xi ' \& a \vee \xi ' \& a \& b \text {. }
$$

Here, $a, b \in\{0,1\}, \quad \xi^{\prime} \geq \xi^{\prime}, ' \quad \xi^{\prime}, \xi^{\prime \prime} \in[0,1]$. Then the expression (4) may be presented as:

$$
\Phi_{\mathrm{V}}=\underset{i=1, l}{\vee}\left(p_{1_{i}} \& p_{2_{i}} \& \ldots \& p_{k_{i}} \& V_{i}\right)
$$

The following property holds: Each disjunctive member in the expression (6) defines the subset of vertices $Y \subseteq X$ with vitality degree $V_{i}$ of fuzzy graph $\tilde{G}=(X, \tilde{U})$. Here subset $Y$ is minimal, in other words, any subset of $Y$ does not have this property.

Considering method works with reachability vertex matrix of first kind fuzzy graph. So in order to apply the method in case of second kind fuzzy graph $\tilde{G}$ we transform this graph into first kind fuzzy graph $\tilde{G}^{\prime}$ like that:

- let $y_{1} \in X$ is the vertex of initial second kind fuzzy graph $\tilde{G}$, and it is adjacent for $t$ vertices and has a degree 
$\mu$. Represent vertex $x$ as directed complete $t$-subgraph of first kind with degree of "inside" edges equals $\mu$.

- connect everyone of the initial "outside" $t$ vertices with one of the vertices of received subgraph.

Example of transformation of second kind fuzzy graph (Fig.1) to first kind fuzzy graph is presented in the Fig.2.

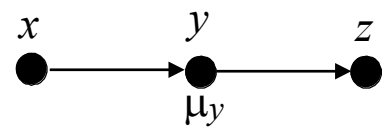

Fig. 1. Initial second kind fuzzy graph.

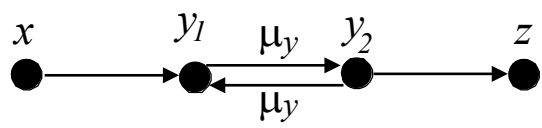

Fig. 2. Transformation to first kind fuzzy graph.

We may prove the next property:

Property. Reachability degree of initial second kind fuzzy graph $\tilde{G}$ coincides with reachability degree of first kind fuzzy graph $\tilde{G}^{\prime}$.

First kind fuzzy graph $\tilde{G}^{\prime}$ comes out of this transformation. Meanwhile, transition from graph $\tilde{G}$ to graph $\tilde{G}^{\prime}$ is biunique. Built reachability matrix $N^{(1)}$ of received first kind fuzzy graph $\tilde{G}^{\prime}$ and pass from it to reachability matrix $N^{(2)}$ of initial second kind fuzzy graph $\tilde{G}$. Apply the foregoing method to the matrix $N^{(2)}$.

Given procedure is considered by example.

Example 1. Let's consider an example of finding of service centers in second kind fuzzy graph $\tilde{G}$ given in the Fig.3.:

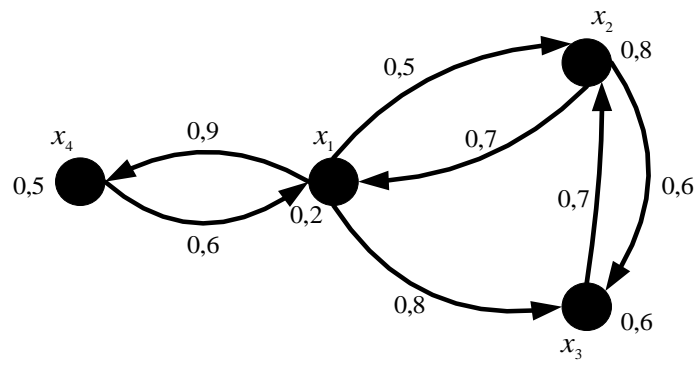

Fig. 3. Second kind fuzzy graph $\tilde{G}$
Here vertices of graph are objects of transport network but edges are paths that connect the objects.

Let's find centers of graph supposing that it is located in graph vertices. In other words service centers should be located in objects of transport network.

The adjacent matrix of fuzzy graph $\tilde{G}$ is presented as:

$$
\begin{array}{c|cccc} 
& x_{1} & x_{2} & x_{3} & x_{4} \\
R_{X} & 0,2 & 0,5 & 0,8 & 0,9 \\
x_{2} & 0,7 & 0,8 & 0,6 & 0 \\
x_{3} & 0 & 0,7 & 0,6 & 0 \\
x_{4} & 0,6 & 0 & 0 & 0,5
\end{array} .
$$

For finding of reachability matrix $N^{(2)}$ of second kind fuzzy graph $\tilde{G}$ we need built new complementary first kind fuzzy graph $\tilde{G}^{\prime}$. This graph is presented in the Fig.4.:

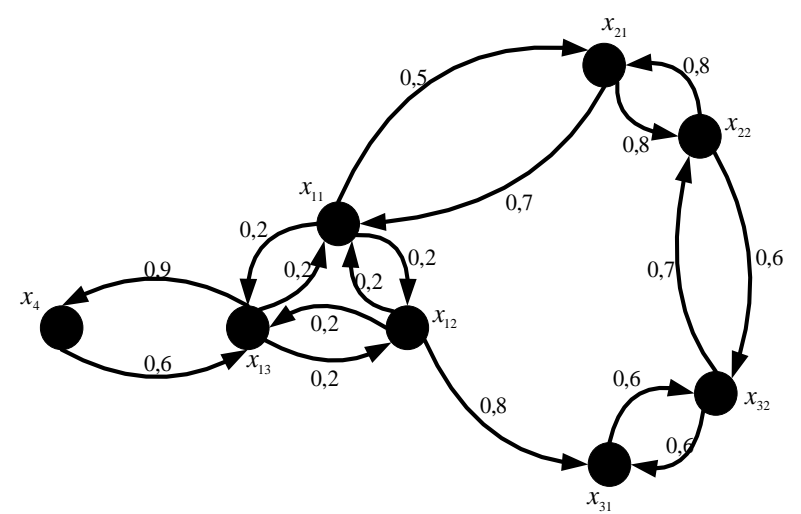

Fig.4. Complementary first kind fuzzy graph $\tilde{G}^{\prime}$.

The reachability matrix $N^{(1)}$ of fuzzy graph $\tilde{G}^{\prime}$ can be presented:

$$
N^{(1)}=\bigcup_{i=0, n-1}^{\bigcup} R_{X}^{i}
$$

Here, $R_{X}^{0}$ - diagonal unitary matrix, $R_{X}^{i}-i$ degree of adjacent matrix. Adjacent matrix of first kind fuzzy graph $\tilde{G}^{\prime}$ takes on form: 


$$
\begin{array}{c|cccccccc} 
& x_{11} & x_{12} & x_{13} & x_{21} & x_{22} & x_{31} & x_{32} & x_{4} \\
x_{11} & 0 & 0.2 & 0.2 & 0.5 & 0 & 0 & 0 & 0 \\
x_{12} & 0.2 & 0 & 0.2 & 0 & 0 & 0.8 & 0 & 0 \\
x_{13} & 0.2 & 0.2 & 0 & 0 & 0 & 0 & 0 & 0.9 \\
R_{X}^{1}=x_{21} & 0.7 & 0 & 0 & 0 & 0.8 & 0 & 0 & 0 \\
x_{22} & 0 & 0 & 0 & 0.8 & 0 & 0 & 0.6 & 0 \\
x_{31} & 0 & 0 & 0 & 0 & 0 & 0 & 0.6 & 0 \\
x_{32} & 0 & 0 & 0 & 0 & 0.7 & 0.6 & 0 & 0 \\
x_{4} & 0 & 0 & 0.6 & 0 & 0 & 0 & 0 & 0
\end{array}
$$

Find degree of matrix $R_{\mathrm{X}}^{2}=R_{X}^{1} \times R_{X}^{1}$, where matrix elements $\quad R_{\mathrm{X}}^{2}=\left\|r_{i j}^{(2)}\right\|, i, j=\overline{1, n}$ are determined as: $r_{\mathrm{ij}}^{(2)}=\underset{k=1, n}{\vee} r_{i k}^{(1)} \& r_{k j}^{(1)}$, where $r_{i k}^{(1)}$ and $r_{k j}^{(1)}$ are elements of matrix $R_{X}^{1}$. Similarly we find $R_{\mathrm{X}}^{\mathrm{i}}=R_{X}^{i-1} \times R_{X}^{1}$, for index $i=3,4,5,6,7$. Let's join the matrixes and find reachability matrix $N^{(1)}=\bigcup_{i=\overline{0,7}} R_{X}^{i}$ :

$$
\begin{aligned}
& \begin{array}{llllllll}
x_{11} & x_{12} & x_{13} & x_{21} & x_{22} & x_{31} & x_{32} & x_{4}
\end{array} \\
& \begin{array}{l|lllllll|l}
x_{11} & 1.0 & 0.2 & 0.2 & 0.5 & 0.5 & 0.5 & 0.5 & 0.2
\end{array} \\
& \begin{array}{l|lllllll|l}
x_{12} & 0.2 & 1.0 & 0.2 & 0.2 & 0.6 & 0.8 & 0.6 & 0.2
\end{array} \\
& \begin{array}{l|llllllll|}
x_{13} & \underline{0.2} & \underline{0.2} & \underline{1.0} & \underline{0.2} & \underline{0.2} & \underline{0.2} & \underline{0.2} & \underline{0.9} \\
\hline
\end{array} \\
& N^{(1)}=x_{21} \quad \mid \begin{array}{llllllllll}
0.7 & 0.2 & 0.2 & 1.0 & 0.8 & 0.6 & 0.6 \mid & 0.2
\end{array} \\
& \begin{array}{l|lll|llll|l}
x_{22} & \underline{0.7} & \underline{0.2} & \underline{0.2} & \underline{0.8} & \underline{1.0} & \underline{0.6} & \underline{0.6} & \underline{0.2}
\end{array} \\
& \begin{array}{l|lll|llllll}
x_{31} & 0.6 & 0.2 & 0.2 & 0.6 & 0.6 & 1.0 & 0.6 & 0.2
\end{array} \\
& \begin{array}{l|llllllll}
x_{32} & \underline{0.7} & \underline{0.2} & \underline{0.2} & \underline{0.7} & \underline{0.7} & \underline{0.6} & \underline{1.0} & \underline{0.2}
\end{array} \\
& \begin{array}{l|llllllll|}
x_{4} & 0.2 & 0.2 & 0.6 \mid & 0.2 & 0.2 & 0.2 & 0.2 \mid & 1.0
\end{array}
\end{aligned}
$$

Each block of complementary matrix conforms to vertex of initial second kind fuzzy graph. So the greatest values are chosen in these blocks. And reachability matrix $N^{(2)}$ of second kind fuzzy graph $\tilde{G}$, given in Fig.2., will be defined as:

$$
\begin{array}{c|cccc}
N^{(2)}= & x_{1} & x_{2} & x_{3} & x_{4} \\
x_{1} & 1 & 0,6 & 0,8 & 0,9 \\
x_{2} & 0,7 & 1 & 0,6 & 0,2 \\
x_{3} & 0,7 & 0,7 & 1 & 0,2 \\
x_{4} & 0,6 & 0,2 & 0,2 & 1
\end{array} \mid .
$$

To construct the expression (6) we rewrite expression (4) like this:

$$
\Phi_{V}=\underset{i=1, n}{\&}\left(a_{i 1} p_{1} \vee a_{i 2} p_{2} \vee \ldots \vee a_{i n} p_{n}\right)
$$

We convert pair $a_{i j} \& p$ from expression (7) to weighted binary vector $a_{i j} \bar{P}_{j}$. Here $\bar{P}_{j}=\left\|p_{i}^{(j)}\right\|$ is a binary vector that has dimension of $n$. The elements of $\bar{P}_{j}$ are defined as:

$$
p_{i}^{(j)}=\left\{\begin{array}{l}
1, \text { if } i=j \\
0, \text { if } i \neq j .
\end{array}\right.
$$

The conjunction of $\left(a_{1} p_{1}\right)$ and $\left(a_{2} p_{2}\right)$ from expression (7) corresponds the conjunction of two weighted binary vectors $a_{1} \bar{P}_{1}$ and $a_{2} \bar{P}_{2}, \bar{P}_{1}=\left\|p_{i}^{(1)}\right\|, \bar{P}_{2}=\left\|p_{i}^{(2)}\right\|, i=\overline{1, n}$, $a_{1}, a_{2} \in[0,1]$. In a vector space the conjunction is defined as $a_{1} \bar{P}_{1} \& a_{2} \bar{P}_{2}=a \bar{P}, \quad$ where $\quad a=\min \left\{a_{1}, a_{2}\right\}, \quad \bar{P}=\left\|p_{i}\right\|$, $p_{i}=\max \left\{p_{i}^{(1)}, p_{i}^{(2)}\right\}, i=\overline{1, n}$.

We define the operation $\leq$ "less or equal" between binary vectors. Binary vector $\bar{P}_{1}$ is less or equal than $\bar{P}_{2}$ if and only if each element of $\bar{P}_{1}$ is less or equal than the corresponding element of vector $\bar{P}_{2}$. Or:

$$
\left(\bar{P}_{1} \leq \bar{P}_{2}\right) \leftrightarrow(\forall i=\overline{1, n})\left[p_{i}^{(1)} \leq p_{i}^{(2)}\right]
$$

Considering the algebra in space of weighted binary vectors, we can make a rule of absorption:

$$
a_{1} \bar{P}_{1} \vee a_{2} \bar{P}_{2}=a_{1} \bar{P}_{1} \text {, if } a_{1} \geq a_{2} \text { and } \bar{P}_{1} \leq \bar{P}_{2} \text {. }
$$

Now we can construct statement (8) using the conjunction operation and the rule of absorption of weighted binary vectors by the following algorithm:

$1^{\circ}$. Each element of the first bracketed expression $(j=1)$ of expression (7) is converted to weighted binary vector. The result is to be written in the first $n$ elements of the buffer vector $\bar{V}_{1}=\left\|v_{i}^{(1)}\right\|, i=\overline{1, n^{2}}$.

$2^{\circ} . j$ incrementing $(j:=j+1)$.

$3^{\circ}$. Each element of the bracketed expression $j$ is also converted to weighted binary vectors. The result is to be written in the first $n$ elements of the buffer vector $\bar{V}_{2}=\left\|v_{i}^{(2)}\right\|, i=\overline{1, n}$.

$4^{\circ}$. The next stage consists of the conjunction of two vectors $\bar{V}_{1}$ and $\bar{V}_{2}$. The result is placed into the buffer vector $\bar{V}_{3}=\left\|v_{i}^{(3)}\right\|, i=\overline{1, n^{2}}$. While placing elements into $\bar{V}_{3}$ , absorption is made using rule (8). 
$5^{\circ}$. All the elements of vector $\bar{V}_{3}$ are copied to vector $\bar{V}_{1}\left(v_{i}^{(1)}:=v_{i}^{(3)}, i=\overline{1, n^{2}}\right)$.

$$
6^{\circ} \cdot j:=j+1 \text {. }
$$

$7^{\circ}$. If $j \leq n$ then goes to $3^{\circ}$, otherwise go to $8^{\circ}$.

$8^{\circ}$. Expression (4) is to be built using elements in the vector $\bar{V}_{1}$. This way we have fuzzy set of vitality of graph $\tilde{G}=(X, \tilde{U})$.

Example 2. The corresponding expression (7) for second kind fuzzy graph $\tilde{G}$, given in the Fig.2, has the next form:

$$
\begin{aligned}
& \Phi_{B}=\left(1 p_{1} \vee 0.6 p_{2} \vee 0.7 p_{3} \vee 0.6 p_{4}\right) \& \\
& \&\left(0.6 p_{1} \vee 1 p_{2} \vee 0.6 p_{3} \vee 0.2 p_{4}\right) \& \\
& \&\left(0.7 p_{1} \vee 0.6 p_{2} \vee 1 p_{3} \vee 0.2 p_{4}\right) \& \\
& \&\left(0.6 p_{1} \vee 0.2 p_{2} \vee 0.2 p_{3} \vee 1 p_{4}\right) .
\end{aligned}
$$

Before the first iteration of the algorithm vectors $\bar{V}_{1}$, $\bar{V}_{2}, \bar{V}_{3}$ and $\bar{V}_{4}$ have the following forms :

$$
\begin{aligned}
& \bar{V}_{1}=\left(\begin{array}{c}
1(1000) \\
0.6(0100) \\
0.7(0010) \\
0.6(0001)
\end{array}\right) \quad \bar{V}_{2}=\left(\begin{array}{c}
0.6(1000) \\
1(0100) \\
0.6(0010) \\
0.2(0001)
\end{array}\right) \\
& \bar{V}_{3}=\left(\begin{array}{c}
0.7(1000) \\
0.6(0100) \\
1(0010) \\
0.2(0001)
\end{array}\right) \quad \bar{V}_{4}=\left(\begin{array}{c}
0.6(1000) \\
0.2(0100) \\
0.2(0010) \\
1(0001)
\end{array}\right) .
\end{aligned}
$$

After the first iteration of the algorithm vector $\bar{V}_{1}$ has the following form:

$$
\bar{V}_{1}=\left|\begin{array}{l}
0.6(1000) \\
1.0(1100) \\
0.6(0100) \\
0.7(0110) \\
0.6(0010) \\
0.2(0001)
\end{array}\right| .
$$

After completing the iterations, finally we have:

Copyright $($ ) Authors

$$
\bar{V}_{1}=\left|\begin{array}{l}
0.6(1000) \\
0.7(1101) \\
1.0(1111) \\
0.2(0100) \\
0.6(0101) \\
0.6(0011) \\
0.2(0001)
\end{array}\right| .
$$

So, the formula (6) for this graph has the form:

$$
\begin{aligned}
\Phi_{V}= & 0.6 p_{1} \vee 0.2 p_{2} \vee 0.2 p_{4} \vee 0.6 p_{2} p_{4} \vee 0.6 p_{3} p_{4} \vee 0.7 p_{1} p_{2} p_{4} \vee \\
& \vee 1 p_{1} p_{2} p_{3} p_{4} .
\end{aligned}
$$

It follows from the last equality that the second kind fuzzy graph $\tilde{G}$ has 7 subsets of vertices with the greatest vitality degree, and fuzzy set of vitality is defined as:

$$
\tilde{V}=\{<0.6 / 1>,<0.6 / 2>,<0.7 / 3>,<1 / 4>\} .
$$

The fuzzy set of vitality defines the next optimum allocation of the service centres: If we have 4 service centres then we place these centres into all vertices. The degree of service equals 1 in this case. If we have 3 service centres then we must place these centres into vertices 1,2 , and 4 . The degree of service equals 0.7 in this case. If we have only one service centre then we must place it into vertex 1 . The degree of service equals 0.6 in last case. Thus, we can conclude that there is no winning when placing two centers.

\section{CONCluSion AND Future Work}

The task of defining of the optimum allocation of centers was considered as the task of the definition of fuzzy vitality set of second kind fuzzy graphs. The algorithm of the definition of fuzzy base set has been proposed. It must be noted; that the considered method makes it possible to define the best service allocations only if the centers are placed in the vertices of a graph (the case of generating new vertices on the edges is not considered). In our future work we are going to examine the problem of the centers' allocation in the temporal fuzzy graphs, i.e. the graphs, edges' membership functions of which change in discrete time.

\section{REFERENCES}

[1] T. Slocum, R. McMaster, F. Kessler, and H. Howard, Thematic Cartography and Geovisualization. 3nd edn. Pearson Education Limited, 2014.

[2] Y. Fang, V. Dhandas, and E. Arriaga, Spatial Thinking in Planning Practice. Portland State University, Portland, 2014.

[3] J. Zhang, and M. Goodchild, Uncertainty in Geographical Information. New York: Taylor \& Francis, Inc., 2002.

ISSN (Print): 2204-0595 
[4] M. Goodchild. "Modelling Error in Objects and Fields". In.: Accuracy of Spatial Databases, M. Goodchild and S. Gopal (eds.) Basingstoke: Taylor \& Francis, Inc., 1989, pp. 107-113.

[5] A. Kaufmann, Introduction a la theorie des sous-ensemles flous. Paris: Masson, 1977.

[6] N. Christofides, Graph theory. An algorithmic approach. London: Academic press, 1976

[7] J. Malczewski. GIS and multicriteria decision analysis. New York: John Willey and Sons, 1999.

[8] I.N. Rozenberg, and T.A. Starostina, Solving of location problems under fuzzy data with using GIS. Moscow: Nauchniy Mir, 2006.

[9] A. Bozhenyuk, and I. Rozenberg. "Allocation of service centers in the GIS with the largest vitality degree," in: Proceedings of the IPMU 2012, Part II, Communications in Computer and Information Science, CCIS 298, Berlin Heidelberg: Springer-Verlag, 2012. pp: 98-106.

[10] V. Bozheniuk, A. Bozhenyuk, and S. Belyakov, "Optimum allocation of centers in fuzzy transportation networks with the largest vitality degree," in: Proceedings of the 2015 Conference of the International Fuzzy System Association and the European Society for Fuzzy Logic and Technology: Atlantis Press, 2015, pp. 1006-1011.

[11] A. Bozhenyuk, S. Belyakov, E. Gerasimenko, and M. Savelyeva. "Fuzzy Optimal Allocation of Service Centers for Sustainable Transportation Networks Service", Intelligent Systems Reference Library, Vol. 113, 2017, pp. 415-437.

[12] J.N.Monderson, and P.S. Nair. Fuzzy Graphs and Fuzzy Hypergraphs. Heidelberg; New-York: Physica-Verl., 2000. 383 p.

L.S. Bershtein, and A.V. Bozhenyuk. "Fuzzy Graphs and Fuzzy Hypergraphs". In: Dopico, J., de la Calle, J., Sierra, A. (eds.) Encyclopedia of Artificial Intelligence, Information SCI, Hershey, New York, 2008, pp. 704-709.

[13] A. Rosenfeld. "Fuzzy graph," in: L.A.Zadeh, K.S.Fu, M.Shimura (Eds.), Fuzzy sets and their applications to cognitive and decision process, New York: Academic Press, 1975, pp. 77 -95.

[14] R.T Yeh, and S.Y. Bang. "Fuzzy relations fuzzy graphs and their applications to clustering analysis," in: L.A.Zadeh, K.S.Fu,
M.Shimura (Eds.), Fuzzy sets and their applications, Academic Press, 1975, pp. $125-149$.

[15] A. Bozhenyuk, I. Rozenberg and D. Yastrebinskaya. "Finding of Service Centers in GIS Described by Second Kind Fuzzy Graphs", World Applied Sciences Journal, Vol. 22(Special Issue on Techniques and Technologies), 2013, pp. 82-86. 